for the first time, demonstrated a large flagellated organism, now known as Trypanosoma evansi-in the blood of horses and camels affected with surra, and this discovery was the pioneer work in the knowledge of protozoan diseases, and has since been of immense benefit to the human race as well as to livestock.

The years between 1860 and 1892 saw considerable advance in the organization of veterinary services in various provinces, and the formation not only of an army veterinary school but also several teaching schools and veterinary colleges. With these must ever be associated as pioneers the names of Hallen, Steel, Pease and Fred Smith; and in most provinces superior veterinary services were formed, officered by members of the Royal College of Veterinary Surgeons with subordinate services officered by graduates of the Indian Veterinary Colleges. The year 1891 marked one of the most important steps ever taken for the development of veterinary work in India, in the appointment of an Imperial bacteriologist; the sequel to which has been the establishment of the well-equipped and well-staffed Veterinary Research Laboratory at Muktesar. It was here that Dr. Lingard, who was the first Imperial bacteriologist, discovered the special affinity of arsenic for the parasite of surra; a discovery which has led to results of greatest importance to the human race. The more recent work of Dr. J. T. Edwards in the production of a goat virus of fixed and reduced virulence for the ox laid the foundation to what has proved a highly successful and economical method of controlling outbreaks of rinderpest amongst plains cattle, without the use of anti-rinderpest serum; the results being to confer a lasting immunity at a fractional cost of the serum methods which had been in vogue previously.
Sir Arthur Olver directed attention to the enormous field for scientific veterinary work which exists in India and deplores the paucity of trained scientific workers, and the heavy loss which could be avoided if more and more scientifically trained staff could be brought into action for combined veterinary and animal husbandry activities. $\mathrm{He}$ suggests that in addition to the five veterinary colleges which at present exist, at three of which the course is only three years, whilst at the other two the course is four, there should be added a Central India University Veterinary College. This would work in conjunction with the Veterinary Research Institutes of Izatnagar and Muktesar to train specially selected students who would be required to undergo a minimum of five and perhaps even six years of scientific veterinary research with special facilities for training in animal husbandry and a university matriculation to enable them to train up to modern standards and to carry out the higher diagnostic and research work which is so essentially necessary to control the epidemics of animals-especially in a tropical country like India, where the wealth and also the health of the people so largely depends on the health of its farm animals. As Sir Arthur said : "the field for such work is unlimited, and up to the present almost un. touched."

The meeting of the Veterinary Section at the Indian Science Congress was well attended, and the papers on veterinary scientific subjects were of a very high order. One notable result ensued in that a proposal to add to future science congresses a Section of Comparative Medicine-in which medical men, veterinarians, physiologists, and those interested in animal husbandry could take equal part, was enthusiastically received and adopted.

\title{
The New Super-Novæ
}

\begin{abstract}
AUMBER of interesting notes on the discovery $A$ and the investigation of the two 'super-novæ', found within the course of a month, by F. Zwicky at the Mount Palomar Observatory, California, have been recently published in the Astronomische Nachrichten.
\end{abstract}

On an earlier occasion Zwicky (Proc. Nat. Acad., $22,459 ; 1936)$ had emphasized the great importance of extensive observation of these unique objects. Super-novm radiate during several weeks as much energy as a whole galaxy of stars, and thus these data may furnish most valuable information on fundamental problems, such as the generation of energy in stars, the evolution of stars and stellar systems, the origin and the characteristics of cosmic rays travelling in space.

The latest discoveries, as well as earlier ones, for example, that in the nebula NGC 4157 in February 1937, are the result of a careful and regular search organized by Hubble, Baade and Zwicky, in the course of which the most important clusters of nebulæ were photographed as often as possible and tens of thousands of nebular images carefully searched for new stars (see Publ. Astro. Soc., Pacific, 49, 290 ; 1937).

Zwicky's recent super-novæ are of special interest because of their enormous absolute brightness, namely, -16 , which makes them the most luminous stars known.
The first of these new stars, "Nova 144.1937 Canum Venaticorum", was found with the 18-in. Schmidt telescope in the faint nebulæ I.C. 4182 on August 31, when it had already reached the magnitude $8 \cdot 5$. It then faded rather rapidly; M. Beyer-Hamburg (Astro. Nach. No. 6307) recorded it on September 2 as $8.85 \mathrm{~m}$., and by September 6 it had decreased to $9.8 \mathrm{~m}$. In this period several spectra have been obtained by K. Walter and W. Strohmeier, of the Astrophysical Observatory at Potsdam (Astro. Nach., No. 6308). The plates show on a rather strong continuous background some very wide emission bands, the whole spectrum having an appearance similar to that of a Wolf Rayet star of early $O \cdot$ type, characterized by bands at $\lambda 4680$ and $\lambda 5411, \mathrm{He}$ II, $\lambda$ 5007, $[0 \mathrm{III}]$, and the Balmer lines. Discussing spectra obtained at the Lick Observatory, D. M. Popper (Publ. Astro. Soc. Pacific, 49, 282 ; 1937) expresses the half-widths of the most distinct bands in $\mathrm{km}$./sec. and finds a velocity of expansion of the star of about $4500 \mathrm{~km}$. $/ \mathrm{sec}$.

The other super-nova was discovered on September 10 in the east end of the nebula NGC 1003. Plates taken at the Hamburg-Bergedorf Observatory on September 6 and September 8 by A. Wachmann showed the star increasing from $13.6 \mathrm{~m}$. to $13 \cdot 3 \mathrm{~m}$. (Astro. Nach., No. 6310). On September 19, W. Strohmeier succeeded in obtaining an objective prism 
spectrum with the Potsdam $30-\mathrm{cm}$. reflector. This plate reveals strong emissions of $\mathrm{He}$ II at $\lambda 5411$ and of the nebular lines of [O III] at $\lambda 5000$, the width being about $200 \mathrm{~A}$. The continuous spectrum resembles that of a $B$-type star. Neutral helium at $\lambda 4471,4388,4144,4026$ appears in absorption. The Balmer lines are present as faint emissions, perhaps with violet absorption companions.

The photographic light curve of this super-nova, "145.1937 Persei", has recently been discussed by A. Beer and C. C. L. Gregory before the Royal Astronomical Society. This paper was based on a number of plates secured with the 24-in. Wilson reflector of the University of London Observatory at Mill Hill. The results show that the brightness of this nova was also fading rapidly. From $13.3 \mathrm{~m}$. on September 20 it decreased to $13.8 \mathrm{~m}$. on September 26 , to $14.6 \mathrm{~m}$. on October $3-4$, and to $15.4 \mathrm{~m}$. on October 9-10. Soon afterwards the star had become too faint for these observations. A high positive colour index is indicated, the visual brightness on October 12 being greater by $1.7 \mathrm{~m}$. than the photographic value. No reports from the large American observatories are yet available, and nothing is known up to now about the doubtlessly very interesting spectroscopic results obtained there.

\section{Development of Co-axial Cables for Television}

$\mathrm{O}^{\mathrm{N}}$ NE of the results of the modern developments in television technique has been the production on a commercial scale of cables capable of trans. mitting, with reasonable efficiency, alternating electric currents at frequencies up to one or two megacjcles per second. These cables, which are of the concentric or co-axial type, are employed in the first place for conveying from the studio or outdoor scene to the television transmitter, the modulation frequency currents of the scene being televised. Such a cable is, for example, employed by the B.B.C. to connect the Alexandra Palace station to Broadcasting House and other important places in central London. But the scope of the application is now being extended to the use of the cables for the interconnexion of television transmitting stations separated by distances of the order of 100 miles. When the distance covered is of this order, the use of the cable is by no means limited to television, since the frequency band avail. able provides a large number of ordinary telephone channels along the cable, thereby considerably augmenting the normal land-line trunk-connexion facilities. By a suitable arrangement of the relative frequencies, these telephone channels can be operated simultaneously with the transmission of the television signals, and the value of the cable is thus considerably enhanced. At the present time, a high-frequency co-axial cable is being installed by the Post Office between London and Birmingham, and the results of its operation will be awaited with interest.

Although Great Britain has been the pioneer country in operating a public television broadcasting service, the development and installation of these cables have been proceeding elsewhere, notably in Germany and the United States. The Wireless World of December 30 contains a note on the work of the German Post Office on a scheme for the linkingup of Berlin with other important towns. The cables in contemplation or actually undergoing installation will provide interconnexion between the television stations; the Post Office will further provide television-telephone facilities and in addition, arrangements have been made for sending as many as two hundred telephone conversations along the new cables in the frequency band not required by the television currents. For the purpose of transmitting television pictures on a basis of 441-line definition and interlaced frames at 25 per second, it will be necessary to instal amplifiers or repeaters at intervals of 10-12 miles along the cable. These repeater stations will be entirely unattended and will be supplied with power-frequency alternating current passed along the cable. Three types of cable made by different firms are being employed, and it is anticipated that the installations will be completed during the current year.

An interesting demonstration of the possibilities of these co-axial cables was provided last November by the transmission of a sound-picture film between New York and Philadelphia, a distance of ninety miles. This matter was briefly referred to in NATURE of December 4 (p. 962), and further details of the experiment have now been supplied by the Bell Telephone Laboratories. The cable in this case consists of two co-axial conductor units, each of which is formed of a flexible copper tube and a single concentric wire insulated at intervals with ebonite disks. Along the route are situated unattended installations of special amplifying equipment which receive their power supply over the two inner conductors. Each of the co-axial conductors with its associated one-way amplifiers is capable of transmitting simultaneously the currents from 240 telephone transmitters. Thus, using the two units of the cable for transmission in opposite directions, the system provides for 240 simultaneous conversations. In the present arrangement, the transmitters are formed into twenty groups of twelve, each transmitter being limited to a frequency band of four kilocycles per second. For the transmission of vision or picture signals, practically the entire frequency band of the cable-about one million cycles per second-is utilized.

In the demonstration to which reference was made above, a cinematograph film moving uniformly past a gate where it was scanned by a rotating disk system at a rate corresponding to 240 lines and 24 frames per second was used to provide a picture. The light passing through the film entered an electron multiplier, and the resulting current contained component frequencies between zero and about 800 kc./sec. For transmission along the cable, the frequency of this signal current was raised by modula. tion about $100 \mathrm{kc}$. $/ \mathrm{sec}$. in order to avoid the portion of the range where transmission would be unsatisfactory and amplification difficult. In passing along the cable, the lowest frequencies fall behind the highest by a time amounting to some twenty microseconds. Delay equalizers were therefore developed to secure equal transmission times for all frequencies 